\title{
GROWTH, UNEMPLOYMENT AND HETEROGENEITY
}

\author{
Piero Ferri, Emeritus Professor \\ Annalisa Cristini \\ AnnaMaria Variato ${ }^{1,2}$ \\ Department of Management Economics and Quantitative Methods \\ University of Bergamo - Italy
}

(August 2018)

\begin{abstract}
The paper analyzes unemployment in a medium-run growth model, where aggregate demand and supply interact. On one hand, autonomous demand drives the dynamics of the system, while heterogeneity in the consumption function, due to the presence of unemployment, strengthens the links with supply aspects. On the other hand, both the rate of growth of labor productivity and labor supply are endogenous. Two major results are obtained. First, unemployment allows the reconciliation between aggregate demand and supply. The second is that unemployment remains bounded and this means that the interaction between aggregate demand and supply thwarts instability. These results are in keeping with those obtained by means of a bottom-up approach, typical of agent based models (ABM). Possible explanations and implications of this convergence are put forward.
\end{abstract}

the study of a nonlinear system where both aggregate demand and supply are endogenous and generate a bounded unemployment, followed by a methodological effort direct to identify possible lines of convergence with the AMB approach. This is a by-product of the presence of heterogeneity in our model.

Key words: bounded unemployment, medium-run growth, endogenous supply, heterogeneity, instability, learning, top-down and bottom-up methodologies.

JEL code: $\mathrm{E} 32, \mathrm{E} 12, \mathrm{~J} 2$

\footnotetext{
${ }^{1}$ We wish to thank two anonymous referees for stimulating suggestions and S. Fazzari (Washington University) for inspiring insights. We also thank the participants to the session of the WEHIA conference at the Catholic University of Milan. Financial support from the University of Bergamo is gratefully acknowledged.

2 Corresponding author: anna.variato@unibg.it
} 


\section{INTRODUCTION}

The post-war literature on growth theory seems to have considered unemployment as a back stage problem. Both the old theories (see Solow, 1956, Kaldor, 1957 and Pasinetti, 1962) and the new growth ones (Romer, 1986) typically assume full employment. When this hypothesis is dropped, the resulting unemployment has two characteristics:

i) it is mainly a supply driven phenomenon;

ii) it is analysed within a search model.

These characteristics are shared by different theoretical models. For instance, Goodwin (1967) and the old neoclassical models typically refer to the first aspect, while more recent contributions, as it is the case of some Schumpeterian approaches, include both. Along this line, Aghion and Howitt (1994) introduce a search model of equilibrium unemployment into a Schumpeterian environment where growth arises from the adoption of new technologies that require labour reallocation for their implementation. The working of the model is based upon two opposite mechanisms that can generate a bounded rate of unemployment. One of these is the capitalization effect, "whereby an increase in growth raises the capitalized returns from creating jobs and consequently reduces the equilibrium rate of unemployment. The other is a creative destruction effect whereby an increase in growth reduces the duration of a job match, thereby raising the equilibrium level of unemployment..." (Aghion and Howitt, 1994, p.477).

The occurrence of the Great Recession has challenged these views leading theoretical developments towards the introduction of alternative tenets. Part of the literature still maintain the above quoted existing set-up examining the effect of extreme conditions (Hall, 2017, for instance, insists on the exogenous role of high discount rates in explaining unemployment considered within a search model). Still a growing set of essays explores the opportunity of more substantive methodological changes. ${ }^{3}$

The view favoured in the present paper is the one subscribing two specific hypothesis (see Ferri, 2011 and 2016). First of all, the analysis will be focused upon a medium-run range (the so called 'intermediate' period in the words of Hyman Minsky, 1982) capable of covering episodes like the Great Recession in their full length. Second, supply (covering both product and labor market) is endogenously settled and in-

\footnotetext{
${ }^{3}$ This literature is embodied and quoted in the three papers we are going to especially emphasize just below.
} 
teracts with aggregate demand; which in turns affects the determinants and the nature of the unemployment rate.

This route is pursued by different articles, but three of them are worth mentioning because they are representative of two different methodological approaches aiming at the same target: Dosi et al. (2010 and 2017) and Fazzari et al. (2018). Both set of authors refer to the endogenous interactions between demand and supply but Fazzari et al. (2018) base their analysis upon a macro approach, while Dosi et al. (2010 and 2017) follow an ABM strategy. Such papers represent respectively the foundation and the challenge of the present analysis.

Indeed the main target of this work is to deepen Fazzari et al. (2018)'s work, and to consider the intersections linking the two approaches. More specifically we study a nonlinear system where both aggregate demand and supply are endogenous and generate bounded unemployment, followed by a methodological effort direct to identify possible lines of convergence with the AMB approach, as a by-product of the presence of heterogeneity in the model. Though the focus may seem narrow, its methodological implications are much richer.

In fact, in order to reach the primary task, three aspects are jointly faced, affecting the structure of the model we are going to discuss. First, unemployment is considered in a medium-run growth perspective enabling the simultaneous relevance of aggregate demand and supply, so to embody the two hypothesis mentioned beforehand. Second, the role of autonomous demand and of consumption heterogeneity between groups of agents are explicitly accounted for in stimulating demand. Finally, the impact is studied of these two features on system stability in the face of the consequent endogeneity of the labour productivity rate of growth (see also Dutt, 2010 and Lavoie, 2011) and labour supply.

The rationale of these choices is multi-faced but organic and unfolds the implicit methodological consequences of this essay devoted to the complexity of macroeconomic dynamics. One deals with the interconnected nature of growth and cycle processes emerging from the interaction between aggregate supply and demand. The specification we are going to suggest would allow to consider jointly the two main Harrod's (1939) problems, i.e. instability and reconciliation (see also Minsky, 1982 and Skott, 2010), if the model generates a bounded rate of unemployment. In other words, the dynamic integrated nature of aggregate demand and aggregate supply would show up through the interaction of two stabilizing levers: on one side, unemployment which represents availability of resources, and in classical terms stands for 
"reserve army", would limit the driver's role of autonomous demand; on the other side, dynamic increasing returns in the supply side would be constrained by aggregate demand. As a result, these two forces could reconcile the rate of growth prevailing in both the product and the labour market, while preventing the system from exploding.

The second choice faces the issue of heterogeneity: it recalls individuals though leaving them into aggregate categories, and it represents an innovation with respect to Fazzari et al. (2018). In the model we are going to show, we have two types of heterogeneity. One acts inside the consumption function affecting different groups of agents, differentiated by their status in the labour market. Such structure is grounded on both micro and macro hypotheses. The micro aspect is that unemployed people have a higher propensity to consume than employed people. The macro aspect is that inequality increases as unemployment rises. This implies that, as unemployment rises, unemployed people possess a lower share of income, then the overall propensity to consume falls when the slack in the labour market increases and this feeds back on the dynamics of the entire system.

The second type of heterogeneity is introduced through the learning process, where individuals form expectations according to the pessimistic vs. optimistic mood of their beliefs. This alternative helps mimicking in a closer way the actual path of the economy.

But the way in which heterogeneity is modelled has a further impact, and creates the organic connection among the arguments of our main target. In fact, its presence makes the product and the labour markets more interconnected so that supply can contribute to stabilize demand beyond the role played by the zero level of unemployment, differently from what happens in Fazzari et al. (2018). Secondly, it helps structuring a top-down model that can naturally be compared with the bottom-up approach put forward by the ABM alternative.

Finally the rationale comes of the third structural choice, i.e. the comparison of the ABM vs. macro approaches; it addresses the issue of the nowadays relevance of aggregate macro models: if proved to be able to give rise to similar expected dynamic paths, and without objecting the role of the first class of models, the compact and intuitive structure of the second class ones would lead to still consider them as a fundamental tool for macroeconomic thinking.

The structure of the paper is the following. Section 2 presents a consumption function based upon heterogeneous groups of agents. Section 3 inserts this equation 
into an integrated nonlinear model based upon endogenous demand and supply. Section 4 considers endogenous supply. Section 5 identifies the steady state of the model. Section 6 illustrates some simulations results where a bounded rate of unemployment emerges. Section 7 presents bifurcation diagrams related to parameters referring to productivity and labour supply. Section 8 considers a process of learning. Section 9 compares these results with those obtained by Dosi et al. (2010) with an ABM approach. Section 10 puts forward some methodological considerations. Section 11 concludes. A final Appendix shows the nonlinear model in a compact way.

\section{HETEROGENEITY AND INEQUALITY IN THE CONSUMPTION FUNCTION}

A strategic starting point in our analysis is the consumption function. With respect to the current literature, it presents two features deserving special attention. On one hand, it considers both durable and non-durable goods. On the other, it introduces the rate of unemployment $\left(u_{t}\right)$ into the picture.

The suggested specification is the following:

$$
C_{t}=\left[Y_{t-1}\left(1+E_{t-1} g_{t}\right)\right]\left[c_{1}-c_{2}\left(u_{t-1}-u_{0}\right)\right]+F_{t}
$$

The term inside the first square parenthesis represents expected income, given by the last period level of income increased by the expected rate of growth. The second square parenthesis shows the propensity to consume non-durable goods, where variables with subscript 0 refer to steady state values. ${ }^{4}$ Finally, $F_{t}$ stands for the consumption of durable goods.

It is legitimate to ask whether the presence of both output and unemployment is meaningful. In other words, it is worth wondering whether one can disentangle the effect of the two variables that in a Keynesian framework are strictly related. In the literature, one can find two different kinds of justification. The first insists on the role of unemployment as a proxy of uncertainty. In this case, higher unemployment implies greater uncertainty and therefore an increase in precautionary saving (and hence a fall in the propensity to consume). This is the line stressed by Carroll (1992), Malley and Moutos (1996) and Palley (2012). The other approach underlines the role that heterogeneity in the employment status can have on the pattern of the overall consumption (see Eusepi and Preston, 2015).

\footnotetext{
${ }^{4}$ Lagged unemployment has been introduced in order to maintain the recursiveness of the model.
} 
This is the alternative chosen in the present model, where wages and prices are given and income distribution is not considered. It follows that one cannot refer to the Kaldorian hypothesis of characterizing the propensity to consume according to the source of income. We rather refer to the employment/unemployment status.

According to Alichi et al. (2016) the polarization between the rich (high income brackets) and poor people (low-income brackets) is a very significant measure of inequality that has two characteristics, at least in the US economy: a) it increased during the Great Recession; b) it impacted on the propensity to consume.

In order to catch these stylized facts, consider the following equation, for the moment referred to non-durable consumption:

$$
C_{t}=C_{t}^{n}+C_{t}^{u}
$$

In other words, total consumption $C_{t}$ is the sum of the consumption of employed $(n)$ and unemployed people $(u)$, whose income is respectively $Y^{n_{t}}$ and $Y{ }_{t}$. Dividing through these incomes, one obtains the different propensities to consume.

In this perspective, the behavior of the overall (average) propensity to consume depends on the pattern of the relative incomes $\left(Y^{u} / Y\right) .^{5}$

In fact, one can write:

$$
\begin{gathered}
\frac{C_{t}}{Y_{t}}=\frac{C_{t}^{n}}{Y_{t}^{n}} \frac{Y_{t}^{n}}{Y_{t}}+\frac{C_{t}^{u}}{Y_{t}^{u}} \frac{Y_{t}^{u}}{Y_{t}} \\
c_{t}=c_{t}^{n} \frac{Y_{t}^{n}}{Y_{t}}+c_{t}^{u} \frac{Y_{t}^{u}}{Y_{t}} \\
c_{t}=c_{t}^{n}\left(1-\frac{Y_{t}^{u}}{Y_{t}}\right)+c_{t}^{u} \frac{Y_{t}^{u}}{Y_{t}} \\
c_{t}=c_{t}^{n}-c_{t}^{n} \frac{Y_{t}^{n}}{Y_{t}}+c_{t}^{u} \frac{Y_{t}^{u}}{Y_{t}}
\end{gathered}
$$

which generate the following expression:

$$
c_{t}=c^{n}+\left(c^{u}-c^{n}\right) \frac{Y_{t}^{u}}{Y_{t}}
$$

Note that equation (2.3) takes as given the propensity to consume for each group. In addition, the following inequality is assumed: ${ }^{6}$

\footnotetext{
${ }^{5}$ According to Eusepi and Preston (2015) the income differential is about $20 \%$ between families belonging to the labor force with respect to those outside. For Hall (2009) the difference is between employed and unemployed and in this case it amounts to $15 \%$. It is worth stressing that these figures were calculated before the Great Recession when unemployment was lower and had a smaller duration.
} 


$$
c^{u}>c^{n}
$$

At this stage of the analysis, another strategic assumption is introduced. Specifically, we assume that the gap between the levels of income of the two groups increases as unemployment rises with respect to its steady state $\left(u_{0}\right)$. It follows that the ratio $\mathrm{Yu}^{\mathrm{u}} / \mathrm{Y}^{\mathrm{n}}$, a measure of income inequality, becomes negatively related to the rate of unemployment $\left(u_{t}\right)$.

In mathematical terms, one can write:

$$
\frac{Y_{t}^{u}}{Y_{t}^{n}}=\eta_{0}-\eta_{1}\left(u_{t}-u_{0}\right)
$$

Inserting (2.4) into (2.3)the following equation is obtained:

$$
c_{t}=c^{n}+\left(c^{u}-c^{n}\right) \eta_{0}-\eta_{1}\left(c^{u}-c^{n}\right)\left(u_{t}-u_{0}\right)
$$

or more simply:

$$
c_{t}=c_{1}-c_{2}\left(u_{t}-u_{0}\right)
$$

which reproduces the second square parenthesis in equation (2.1), where

$$
c_{1}=c^{n}+\left(c^{u}-c^{n}\right) \eta_{0}
$$

and

$$
c_{2}=\left(c^{u}-c^{n}\right) \eta_{1}
$$

An increase in $u_{t}$ decreases the propensity to consume because the inequality effect shrinks unemployed income in the presence of a greater rate of unemployment.

Some qualifications are to be introduced. First of all, heterogeneity is referred to two groups of agents, i.e. unemployed versus employed people. It is therefore different from the concept used by the ABM approach. In the second place, unemployment is a proxy of poverty. However, and this is the third aspect, the origin of inequality is unexplained, since only its changes are taken into consideration. Fourthly, in this context, inequality changes are accounted for by macro variables, ${ }^{7}$ which impact on the overall propensity to consume. This is the final aspect that closes the loop.

\footnotetext{
${ }^{6}$ Alichi et al. (2016) confirm this inequality referring to the different bracket of income. Since unemployment is a source of poverty, this indirectly justifies the above assumption.

7 This strategy of referring to macro variables as indicators of inequality is discussed in Ferri (2016).
} 


\section{A NONLINEAR MODEL}

This consumption function based upon heterogeneous agents is to be inserted within the tenets of a nonlinear model (see Fazzari et al., 2018) To this purpose, a preliminary aspect to be considered is the information structure and the formation of expectations. Suppose, initially, that expectations of the rate of growth $\left(g_{t}\right)$ are formed according to the following adaptive rule:

$$
E_{t-1} g_{t}=(1-\alpha) g_{t-1}+\alpha E_{t-2} g_{t-1}
$$

(see Ferri and Variato, 2010 and Ferri, 2011 for more sophisticated formulae) where $\mathrm{E}_{\mathrm{t}-1}$ is the expectation operator at time $\mathrm{t}-1 .{ }^{8}$

While the consumption function is replicated for ease of reading,

$$
C_{t}=\left[Y_{t-1}\left(1+E g_{t}\right)\right]\left[c_{1}-c_{2}\left(u_{t-1}-u_{0}\right)\right]+F_{t}
$$

$\mathrm{F}_{\mathrm{t}}$, the consumption of durable goods, is represented in the following way:

$$
F_{t}=F_{t-1}\left(1+g^{*}\right)\left[1-\psi_{1}\left(u_{t-1}-u_{0}\right)\right]
$$

where $\mathrm{g}^{*}$ is an exogenous rate of growth of durable consumption, while $\psi_{1}$ measures the correction induced by the presence of unemployment in excess of steady state.

Three aspects deserve some attention. The first one is that in this case unemployment can be interpreted as a proxy of uncertainty (see Malley and Moutos, 1996). The second is that in the steady state its role vanishes. This implies that durable goods assume the status of autonomous demand, which drives the system. ${ }^{9}$

In order to determine investment, consider the following equation that states that the evolution of aggregate demand stimulates the following desired capacity:

$$
K_{t+1}=v^{*} E Y_{t+1}=v^{*}\left(1+E g_{t}\right)^{2} Y_{t-1}
$$

Where $\mathrm{K}$ is capital, while $\mathrm{v} *$ is the desired capital- output coefficient.

In this context, investment plays different roles: $\mathrm{i})$ it replaces depreciation $(\delta)$; ii) it accounts for steady state growth in desired capacity $\left(E g_{t} K_{t}\right)$ and iii) at least partially it closes the gap between actual and desired capacity, as appears from the following equation, where the three components are clearly stated:

\footnotetext{
${ }^{8}$ Henceforth, the time dimension of $\mathrm{E}$ will be dropped. It will be resumed only when dealing with learning in Section 8.

${ }^{9} \mathrm{Ft}$ can also represent other types of autonomous demand, such as public expenditure or exports (see Lavoie, 2014).
} 


$$
I_{t}=\delta K_{t}+E g_{t} K_{t}+\beta\left[v^{*}\left(1+E g_{t}\right)^{2} Y_{t-1}-\left(1+E g_{t}\right) K_{t}\right]
$$

where $\beta$ represents the speed of adjustment of actual investment with respect to the desired one.

In this context, the process of actual capital accumulation is fed by a lagged investment, which reflects production lags:

$$
K_{t}=(1-\delta) K_{t-1}+I_{t-1}
$$

Aggregate demand is defined as the sum of consumption and investment:

$$
Y_{t}=C_{t}+I_{t}
$$

It has to face aggregate supply. The way in which this happens contributes to determine both the nature and the properties of the system.

\section{AN ENDOGENOUS NATURAL RATE OF GROWTH}

The level of output supply $\left(Y_{t}^{S}\right)$ is specified in the following dynamic form:

$$
Y_{t}^{s}=Y_{t-1}^{s}\left(1+g_{t}^{S}\right)
$$

Where $\mathrm{g}^{\mathrm{s}}$ represents what Harrod named the natural rate of growth. It can be defined in the following manner:

$$
g_{t}^{S}=\left(1+\tau_{t}\right)\left(1+\sigma_{t}\right)-1
$$

In other words, it depends on the rate of productivity growth $\left(\tau_{t}\right)$ and the rate of growth of labor supply $\left(\sigma_{t}\right)$, plus an interaction term. In the present model, both labor supply and productivity are to be determined endogenously. Consider first labor supply that can be expressed by the following equations:

$$
\begin{gathered}
L_{t}=\left(1+\sigma_{t}\right) L_{t-1} \\
\sigma_{t}=\rho_{0}-\rho_{1} u_{t-1}
\end{gathered}
$$

These equations imply that the dynamics of labor supply is endogenous and its rate of change depends negatively on the rate of unemployment. It is worth stressing that there are two distinguishing features with respect to the current literature. Labor supply is not a mere demographic phenomenon, but depends on such factors as migrations and labor participation that are sensitive to economic conditions (see Delong and 
Summers, 2013). In particular, the IMF (2018) has shown the negative role that unemployment can have on labor supply. It follows, and this is the second aspect, it allows to overcome the dichotomy between fixed and infinitively elastic supply, the former typical of advanced countries and the latter characterizing the developing ones. Likewise, output per man $\left(A_{t}\right)$ can be expressed in an analogous way:

$$
A_{t}=\left(1+\tau_{t}\right) A_{t-1}
$$

Where $\tau$ is assumed to depend on the accumulation rate:

$$
\tau_{t}=\theta_{0}-\theta_{1} \frac{i_{t-1}}{v_{t-1}}
$$

Let the investment ratio and the actual capital-output ratio be respectively defined as follows:

$$
\begin{gathered}
i_{t}=\frac{I_{t}}{Y_{t}} \\
v_{t}=\frac{K_{t}}{Y_{t}}
\end{gathered}
$$

One of the most important determinants of the rate of growth of productivity is investment, as Kaldor (1978) stressed long-time ago and as the recent literature on investment on $R \& D$ has shown. The specification (4.6) is in line with the KaldorVerdoorn law. As McCombie (2002, p.99) underlines: "the basis of the Verdoorn Law would seem to be a linear Kaldorian technical progress function with an allowance for increasing returns".

In the present perspective, the gross rate of accumulation, which includes depreciation, becomes the strategic factor. It is simply equal to the investment ratio divided by the capital-output ratio. In order to close the labor market, the demand for labor and the rate of unemployment must be included. They are equal respectively to:

$$
N_{t}=\frac{Y_{t}}{A_{t}}
$$

and

$$
u_{t}=1-\frac{N_{t}}{L_{t}} \geq 0
$$

Finally, the rate of growth of demand is defined as: 


$$
g_{t}=\frac{Y_{t}}{Y_{t-1}}-1
$$

Given an exogenous rate of autonomous demand growth $\mathrm{g}^{*}$ and the expected-desired capital-output ratio $v^{*}$, the system refers to 17 unknowns: $I_{t}, K_{t}, C_{t}, F_{t}, Y_{t}, u_{t}, N_{t}, L_{t}, g_{t}$, $\mathrm{V}_{\mathrm{t}}, \tau_{\mathrm{t}}, \mathrm{A}_{\mathrm{t}}, \sigma_{\mathrm{t}}, \mathrm{i}_{\mathrm{t}}, \mathrm{Eg}, \mathrm{Y}_{\mathrm{t}}^{\mathrm{s}}$ and $\mathrm{g}_{\mathrm{t}}^{\mathrm{s}}$. They are inserted into 17 equations, ranging from (3.1) to (3.6) and from (4.1) to (4.11).

\section{STEADY STATE AND THE RECONCILIATION PROCESS}

In steady state all rates of growth must equal. In this perspective, it is convenient to express the Harrodian natural rate of growth by substituting equations (4.4) and (4.6) into (4.2):

$$
g_{t}^{S}=\left(\rho_{0}+\theta_{0}+\rho_{0} \theta_{0}\right)+\theta_{1} \frac{i_{t-1}}{v_{t-1}}+\rho_{0} \theta_{1} i_{t-1}-\left(\rho_{1}+\rho_{1} \theta_{0}+\rho_{1} \theta_{1} \frac{i_{t-1}}{v_{t-1}}\right) u_{t-1}
$$

A fixed natural rate of growth is represented by the terms included in the first parenthesis, which are simply the compounding of a fixed rate of growth for labor supply $\left(\rho_{0}\right)$ and productivity $\left(\tau_{0}\right)$. Another aspect to be considered is the presence of the rate of unemployment.

Unemployment in the model serves two purposes. On one hand, it can justify the assumption of given wages and prices. In the second place, it can facilitate the process of reconciliation between aggregate demand and supply as will be shown shortly. In other words, unemployment plays the classical role of "reserve army" providing resources to the system. Steady state for demand ( 0 used as a subscript refers to a steady state situation) implies that: ${ }^{10}$

$$
g_{t}=g^{*}
$$

and

$$
i_{0}=v^{*}\left(g^{*}+\delta\right)
$$

In other words, demand must grow at the same rate of autonomous demand and the investment ratio must correspond to the "warranted" rate. Furthermore, $f_{0}$, i.e. the

\footnotetext{
10 This is derived from equation (3.3), when both sides are divided by $Y_{t}$ and the steady state
} conditions are imposed. 
steady state share of autonomous demand is endogenously given by: ${ }^{11}$

$$
f_{0}=\left(1-c_{1}\right)-v^{*}\left(g^{*}+\delta\right)
$$

These equations underline the Keynesian nature of the model because investment drives saving. Furthermore

$$
g_{0}=g_{0}^{s}
$$

implies (from 6.2) that

$$
g^{*}=\rho_{0}+\theta_{0}+\rho_{0} \theta_{0}+\theta_{1}\left(g^{*}+\delta\right)+\rho_{0} \theta_{1}\left(g^{*}+\delta\right)-\left[\rho_{1}+\rho_{1} \theta_{0}+\rho_{1} \theta_{1}\left(g^{*}+\delta\right)\right] u_{0}
$$

and that

$$
u_{0}=\frac{\left[\rho_{0}+\tau_{0}\left(1+\rho_{0}\right)\right]-g^{*}}{\rho_{1}\left(1+\tau_{0}\right)}
$$

The values of the variables and of the parameters must be such to generate the usual inequality, $u_{0} \geq 0$.

The rate of unemployment depends on both demand and supply factors. In fact, $\mathrm{u}_{\mathrm{t}}$ depends on the value of the driver of the system, i.e. $\mathrm{g}^{*}$ but also on the parameters of the labor supply and productivity equations. An increase in the parameters of $\tau_{0}$ makes $\mathrm{u}_{0}$ change in the same direction, while the impact of $\rho_{0}$ and $\rho_{1}$, which pertain to labor supply, is of opposite sign: positive the former, negative the latter. Finally, one has to ascertain their dynamic impact on the working of the system.

\section{BOUNDED UNEMPLOYMENT}

The nonlinear system has been simulated (A compact description of the system is illustrated in the Appendix). The values of the parameters are shown in Table 1. These values are within the range found in the empirical literature as discussed by Fazzari et al. (2018).

(The value of $\rho_{0}$ has been calibrated so to generate a steady state value of unemployment equal to $5 \%$ ).

11 This is derived from equation (3.6), divided both sides by $Y_{t}$ and substituting equation (3.2) and (4.7). 
Given the values of the parameters, the steady state of the model has been shocked for one period. ${ }^{12}$ The ensuing dynamics are illustrated in Figure 1, where the last 50 values out of 1000 runs are shown.

Table1. The values of the parameters

\begin{tabular}{|c|c|c|}
\hline $\mathrm{c}_{1}=0.62$ & $\delta=0.12$ & $\mathrm{v}^{*}=0.808$ \\
\hline $\mathrm{c}_{2}=0.30$ & $\mathrm{~g}^{*}=0.04$ & $\beta=0.1$ \\
\hline$\Psi 1=0.1$ & $\rho_{0}=0.025$ & $\rho_{1}=0.7$ \\
\hline$\theta_{0}=0.005$ & $\theta_{1}=0.20$ & $\alpha=0.8$ \\
\hline
\end{tabular}

Three observations are worth stressing at this stage of the analysis. The first is that the model presents persistent fluctuations. In the second place, fluctuations are always bounded and this is the result of the interplay between aggregate demand and supply.

Figure 1: The dynamics of the model
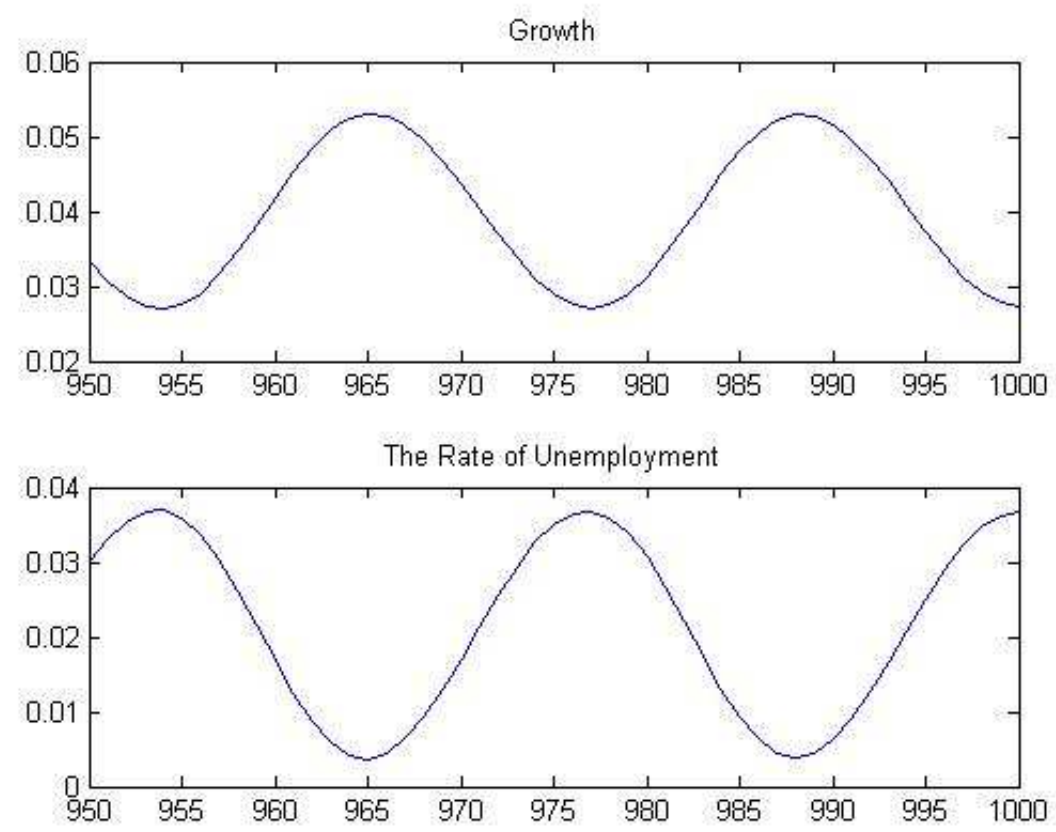

The mechanisms behind these results are quite simple and straightforward. The driving force of the model is a super-multiplier (see Hicks, 1950, for a definition) ${ }^{13}$ accompanied by the presence of a stock-adjusting mechanism in the investment func-

\footnotetext{
12 Alternatively, one can chose an initial period different from the steady state.

13 Briefly, it refers to the presence of autonomous demand along with the traditional determinants of the multipliers.
} 
tion. When the steady state of the system is shocked, for example, by events that affect expectations, aggregate demand depresses the rate of growth and makes unemployment increase. However, the presence of a growing autonomous demand can stop this process and transform it into a virtuous circle, reinforced by the(decreasing) inequality feedback. On its turn, this ascending phase is checked by the presence of two supply feedbacks.

It is the presence of these feedbacks that makes the correlation between growth and unemployment a complex one. In fact, if it is true that an increase in $\mathrm{g}$ makes the rate of unemployment decrease, it is also true that an increase in the rate of growth stimulates productivity and affects labor supply. The relative weight of these two forces can cause an increase in the rate of unemployment that, via the inequality feedback, can negatively affect consumption.

Finally, the dynamic profile is not enough complex to meet the challenge of the mimicking actual economies.

\section{SENSITIVITY ANALYSIS}

Two aspects of the obtained results deserve some more attention. The first one is that they are obtained without violating the inequality $u_{t}>0$. This circumstance depends on the value chosen for the parameters. The second one is that the dynamics of the model are very similar to those obtained by Fazzari et al. (2018). The two models are very similar and so the result should not be surprising. In fact, there are only three differences; in Fazzari et al. (2018), $\beta=1$ in the investment equation (3.4), while in the consumption function $\mathrm{c}_{2}$ and $\psi_{1}$ are set equal to zero.

The latter assumption, however, has deeper implications than it might appear at first sight. In fact, the presence of one of the two hypotheses in the consumption function, for instance $c_{2}>0$, implies that the supply aspects become more integrated with the demand side. Their coefficient have an impact on the overall stability of the system, as appears from Figure 2.

(The emphasis has been put on the parameters characterizing productivity and labor supply, i.e. $\theta_{1}$ and $\left.\rho_{1}\right)^{14}$.

The endogeneity of both labor supply and productivity not only contributes to reconcile in equilibrium the rate of growth of output (the so called warranted rate of growth) with that emerging from the labor market (the so called natural rate) but also

\footnotetext{
${ }^{14}$ The bifurcation diagrams relative to the other parameters are in keeping with the results obtained by Fazzari et al. (2018).
} 
helps thwarting instability, as emerges from Figure 2. Furthermore, it is worth considering that $\rho_{1}$ has a greater area of instability with respect to $\theta_{1}$, the parameter reflecting the flexibility of productivity growth.

Figure 2: The dynamic impact of higher $\theta_{1}$ and $\rho_{1}$

(The rate of unemployment is on the $y$-axis)

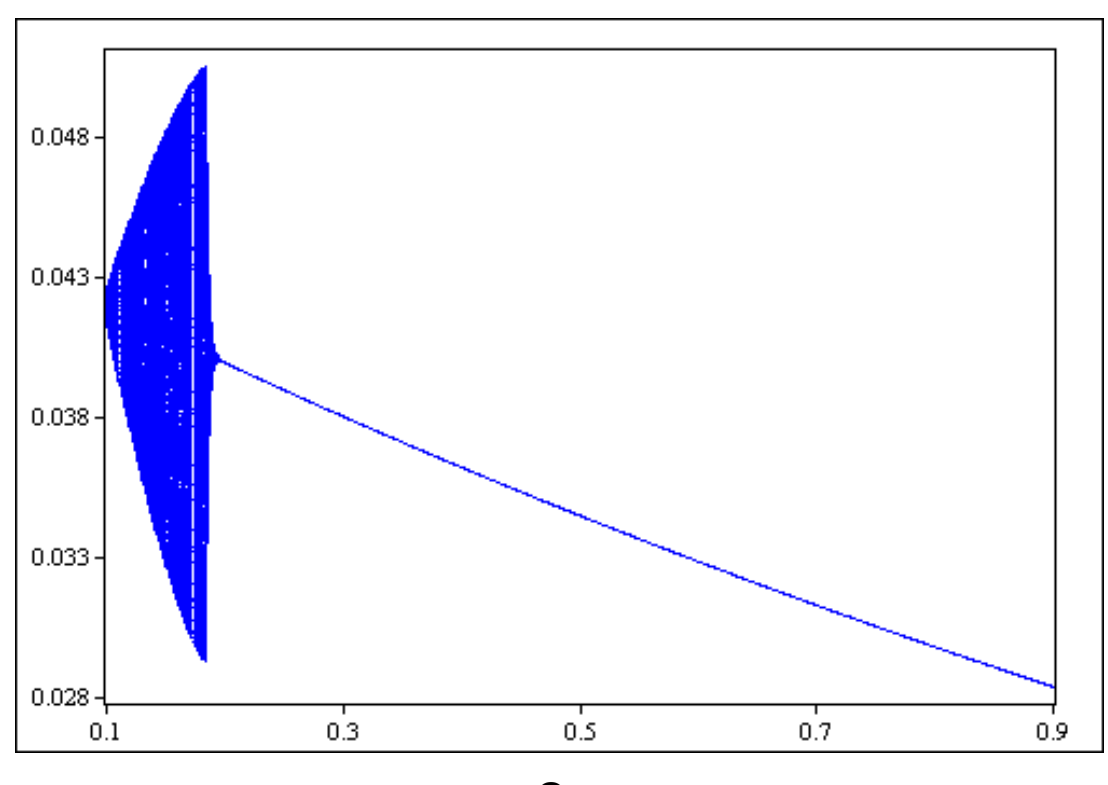

$\Theta_{1}$

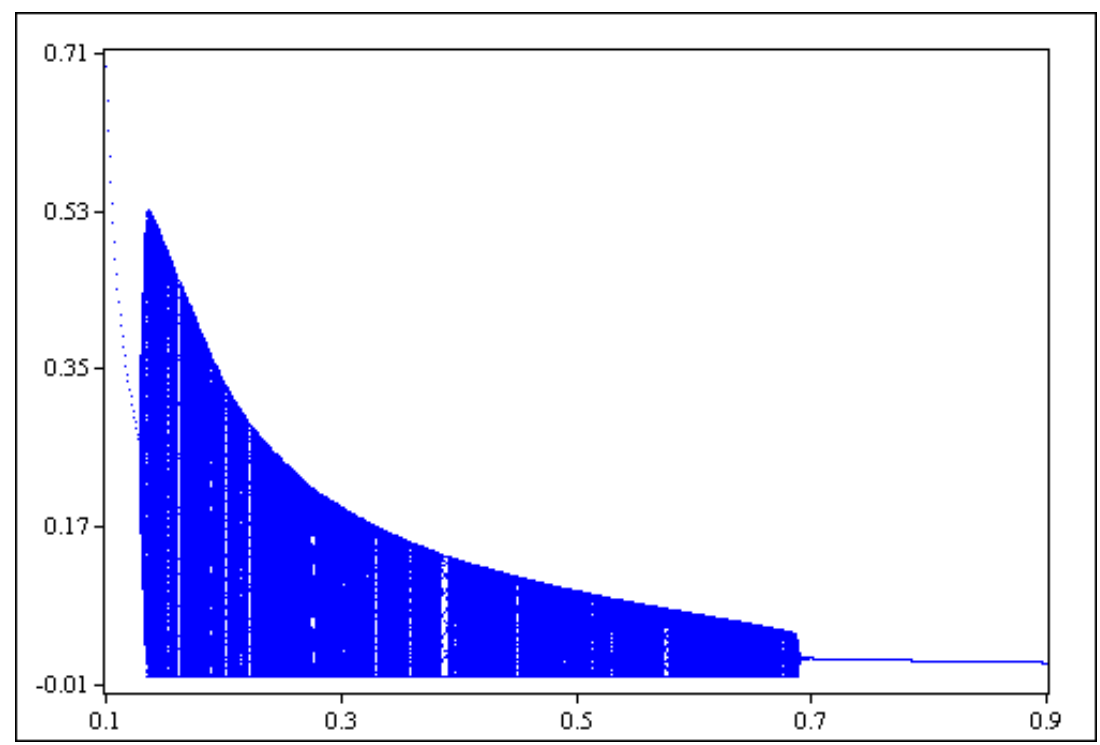

$\boldsymbol{\rho}_{1}$

One important lesson can be learned from the above analysis. The scrutiny of the dynamic pattern generated by a model is not enough to capture its deeper nature. As is well known, the consideration of its stability property is an essential part of the analysis. 


\section{LEARNING WITH HETEROGENEOUS BELIEFS}

One might wonder whether the results depend on the hypothesis made about expectations in an essential way. In other words, it is legitimate to ask whether adaptive expectations are a condition sine qua non in order to obtain endogenous fluctuations in the present model.

In what follows, the adaptive formula of expectations is replaced by a process of learning. The number of learning devices is really numerous so that one cannot pretend to be exhaustive. Let us assume that agents do not have a complete knowledge of the model and therefore use simple rules to forecast the future output growth. We suppose that there are heterogeneous beliefs and that, as is done in De Grauwe (2011), the agents can be either optimistic or pessimistic. ${ }^{15}$ The optimists' forecast is given by the following relationship:

$$
\bar{E}_{t}^{o p t} g_{t+1}=\xi g_{0}+(1-\xi) g_{t-1}
$$

If $\xi=0$ they expect that the steady state rate of growth is always prevailing. On the other hand, the pessimists forecast a smaller rate:

$$
\bar{E}_{t}^{p e s s} g_{t+1}=\eta \bar{E}_{t}^{o p t} g_{t+1}
$$

where $\eta$ is lower than $|1|$ because it can be negative.

The market forecast is obtained as a weighted average of these two forecasts:

$$
\begin{aligned}
& \bar{E}_{t} g_{t+1}=\alpha_{o p t, t} \bar{E}_{t}^{o p t} g_{t+1}+\alpha_{\text {pess }, t} \bar{E}_{t}^{\text {pess }} g_{t+1} \\
& \text { where } \alpha_{o p t, t}+\alpha_{\text {pess }, t}=1
\end{aligned}
$$

Following Brock and Hommes (1997), a selection mechanism is introduced. In fact, agents compute the forecast performance by referring to the mean squared forecasting error:

$$
\begin{gathered}
U_{o p t, t}=-\sum_{k=1}^{\infty} \chi_{k}\left[g_{t-k}-\bar{E}_{\text {opt }, t-k-1} g_{t-k}\right]^{2} \\
U_{\text {pess }, t}=-\sum_{k=1}^{\infty} \chi_{k}\left[g_{t-k}-\bar{E}_{\text {pess }, t-k-1} g_{t-k}\right]^{2}
\end{gathered}
$$

Where $\chi$ represents geometrically declining weights.

\footnotetext{
15 This formulation is well known in finance. Dieci and He (2018) name it "HAM" i.e. heteroge-
} neous agent model. 
The proportion of agents are determined à la Brock and Hommes (1997):

$$
\begin{aligned}
& \alpha_{o p t, t}=\frac{\exp \left(\gamma U_{o p t, t}\right)}{\exp \left(\gamma U_{o p t, t}\right)+\exp \left(\gamma U_{\text {pess }, t}\right)} \\
& \alpha_{\text {pess }, t}=\frac{\exp \left(\gamma U_{\text {pess }, t}\right)}{\exp \left(\gamma U_{\text {opt }, t}\right)+\exp \left(\gamma U_{\text {pess }, t}\right)}
\end{aligned}
$$

Where $\gamma$ measures the intensity of choice. These formulae indicate that those that had a success in the past will convince more people to follow them in the future. ${ }^{16}$ The parameters of the new model are included in Table 2.

Table 2. The values of the parameters in the stochastic case with learning

\begin{tabular}{|c|c|c|}
\hline $\mathrm{c}_{1}=0.62$ & $\delta=0.12$ & $\mathrm{v}^{*}=0.95$ \\
\hline $\mathrm{c}_{3}=0.20$ & $\mathrm{~g}^{*}=0.02$ & $\beta=0.5$ \\
\hline$\Psi_{1}=0$ & $\rho_{0}=0.07$ & $\rho_{1}=0.7$ \\
\hline$\theta_{0}=0.005$ & $\theta_{1}=0.20$ & \\
\hline$\rho_{\text {learning }}=0.2$ & $\chi_{\text {learning }}=10000$ & $\xi=-0.2$ \\
\hline
\end{tabular}

while the dynamics of the model are illustrated in Figure 3.

Figure 3: The Dynamics with learning and productivity shocks
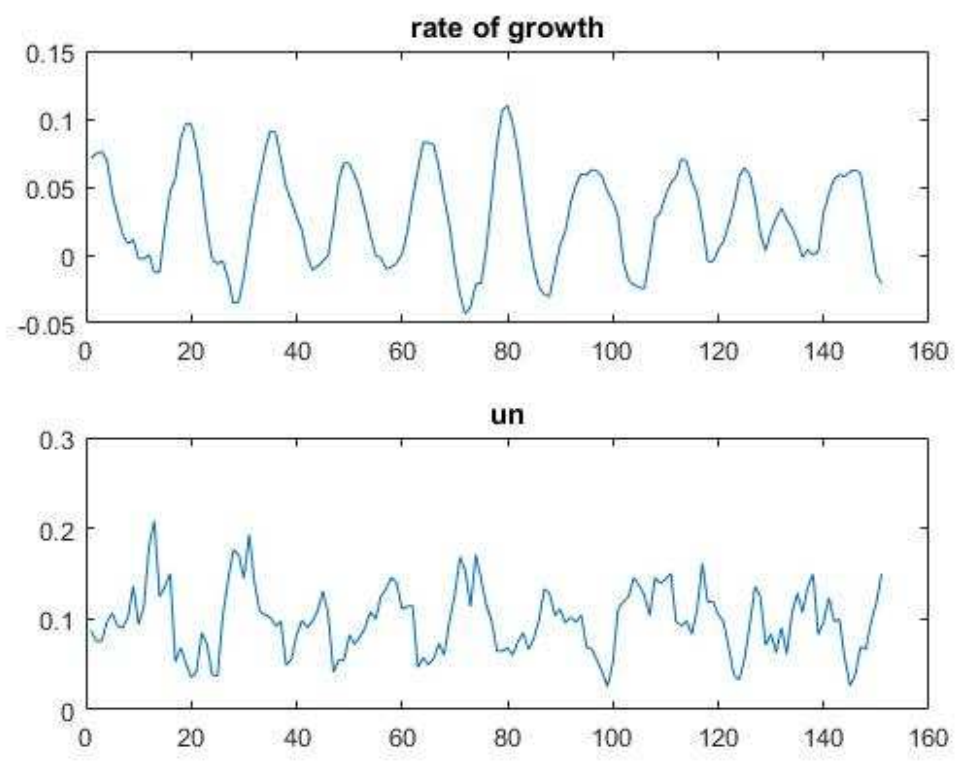

16 This selection mechanism can be interpreted as an evolutionary one, as stressed by De Grauwe (2008). 
Figure 4: The sensitivity analysis for $\theta_{1}$ and $\rho_{1}$

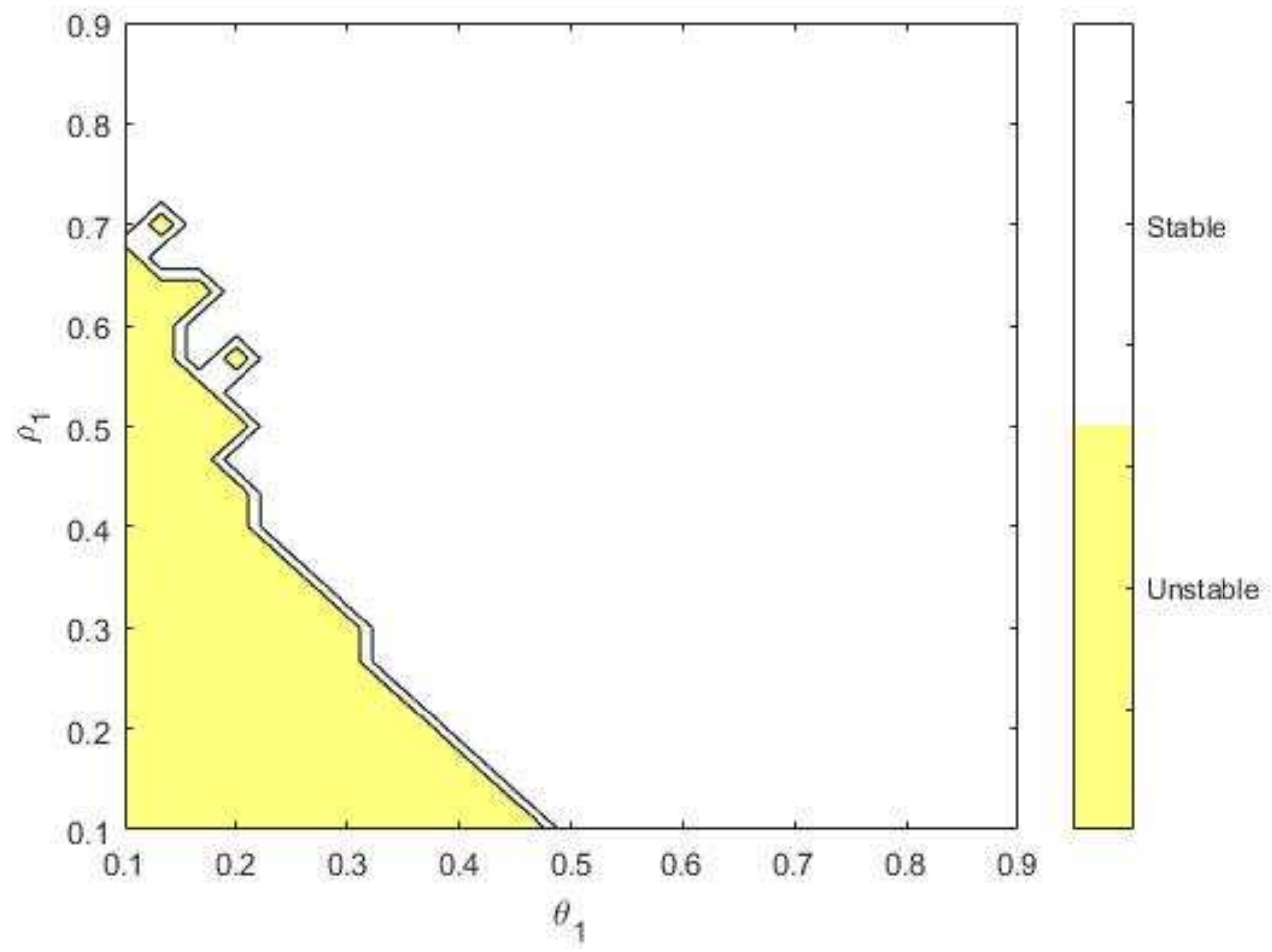

As one can see from Fig. 4 , the values of both $\rho_{1}$ and $\theta_{1}$ leading to instability are in keeping with the results emerging from Fig. 2.

\section{A SKETCHY COMPARISON WITH AN ABM MODEL}

From a methodological point of view, the macro approach that has produced these results can be defined "top down", where the second adverb is justified by the presence of heterogeneous consumers. If one wants to consider a forerunner of this approach, Minsky is certainly one of the most important as his posthumous published dissertation (see Minsky1954-2004) witnesses.

In dealing with the foundations of business cycles, Minsky focused the attention upon the nature of the equations characterizing a model (and in particular, the investment function). In this case, the parameters of the functions included in the aggregative model are to be interpreted as short symbols for more complex processes. In other words, one has to construct a model consisting of an inner circle of relationship between the most important macroeconomic variables and a series of supplementary relationships directed at studying these macro variables.

Two are the main characteristics of this approach. The first is that the macro coefficients are the result of the sum of disaggregated levels. The second is that these val- 
ues reflects a complex micro analysis referring to behavior of firms, functioning of the markets and financial conditions.

Our approach is close to this formulation if one replaces investment with consumption. As it has been anticipated, heterogeneity in consumption is of a special nature. It refers to two groups of people. It would be tempting, at this stage of the analysis, to make a comparison with the so called agent-based models ( $A B M)$, where heterogeneity refers to $\mathrm{n}$ agents. Since it is beyond the scope of the present model to deepen these methodological differences, ${ }^{17}$ we choose the simpler strategy of finding areas of intersections.

Dosi et al. (2010) is certainly very appropriate for the task we intend to pursue. Their ABM model is based upon the interaction between Schumpeterian and Keynesian aspects, capable of generating an endogenous growth model with bounded unemployment. They have developed other models along these lines (2017). However, we prefer to focus the attention on their 2010 paper because it has a structure closer to ours, even though our supply aspects are more black-boxed, to use their expression, with respect to their Schumpeterian approach, where heterogeneity is a structural aspect.

To this purpose, let us consider Table 3, where the results related to the strategic variables, $\mathrm{g}_{\mathrm{t}}$ and $\mathrm{u}_{\mathrm{t}}$, are taken into consideration for both models. ${ }^{18}$

Table 3: Comparing the results of two different models

\begin{tabular}{|l|c|c|}
\hline & Our model & Dosi et al. (2010): Table 7 \\
\hline Average g & $\begin{array}{c}0.0270 \\
(0.0494)\end{array}$ & $\begin{array}{c}0.0254 \\
(0.0809)\end{array}$ \\
\hline Unemployment rate & $\begin{array}{c}0.0929 \\
(0.0368)\end{array}$ & 0.1072 \\
\hline (standard dev) & -0.60 & -0.66 \\
\hline
\end{tabular}

The comparison is made with their benchmark scenario. The similarities are remarkable, even though this is more true for the average values than for the standard devia-

\footnotetext{
17 See Ferri (2019) for a deeper discussion on these methodological aspects.

18 Dosi et al. (2010) have a richer time series analysis that is not considered because our focus is on growth and unemployment.
} 
tions. In order to obtain similar values for the standard deviation, one has to refer to a scenario characterized by a strong antitrust policy. In this case, it would assume the value of $0.05 .{ }^{19}$

The contents of Table 3 are only a first step in the comparison of dynamic models. They are necessary but not sufficient steps in order to draw strong conclusions. The same dynamic pattern, as has been already shown, can be compatible with different model structures and with opposite stability properties. This is true within the ABM models (see Dawid and Delli Gatti, 2018) and it is a fortiori true for models referring to different methodologies.

It follows that further investigation is needed in order to catch the intersection between two sets of methodologies that seem in opposition between them.

\section{METHODOLOGICAL CHALLENGES}

According to Delli Gatti et al. (2014) the ABM approach can be qualified in the following manner:" ...borrowing concepts from the science of complexity, we rationalize market economies as complex adaptive systems, where 'bounded-rational agents' micro decisions produce the emergence of unintended aggregate outcomes, which in turn feedback on the individual behavior (downward causation)."(p. 117)

This approach is in strong contrast with the traditional macroeconomics where a very sophisticated individual behavior is linked together by simple macroeconomic structure. In this context, there is an isomorphism between micro results and macro aspects which disappears in the ABM model, where simple micro behavior is inserted into a sophisticated environment (see also Judd and Tesfatsion, 2006).

Since the analysis of the present paper cannot be represented by the above description of a macro approach, it deserves other kinds of considerations.

Dosi et al. (2010) refer essentially to a bottom-up approach, where the macro results depend on the micro interactions among heterogeneous agents. In this environment, supply aspects are modeled through a Schumpeterian approach, while aggregate demand is fundamentally Keynesian. Our approach is fundamentally macro, with a more developed aggregate demand and an endogenous supply that is more oriented to the dynamic economies of scale. However, it refers also to the presence of heterogeneous groups of people, the employed and the unemployed that govern changes in inequality and that impact on aggregate demand through changes in the

${ }^{19}$ In our model, an increase in beta or in $\mathrm{c}_{3}$ would increase the standard deviations of both $\mathrm{g}$ and $\mathrm{u}$. 
propensity to consume. In this sense, our approach is top down with feedback effects on macro variables.

Despite their differences, there are, however, also similarities between the two approaches that deserve to be identified. First of all, they both refer to bounded rational agents, make use of nonlinearities and consider the analysis in a state of disequilibrium. In the second place, they both have a complete feedback loop. The bottom-up approach has a feedback going into the opposite direction (the so called downward causation), while the top-down has a feedback going upward. In other words, they both implement a two-way approach to the micro-macro relationships. Secondly, this common background generates macro results that reflect interactions between markets and individuals and therefore are not isomorphic to the representative agent. Finally, we refer to a learning process that is based upon a heterogeneous agent model and that, according to Dieci and He (2018) has "strong connections with a broader area of Agent-Based Models (ABMs) and Agent-based Computational Economics (ACE)" (p.259).

For all these reasons, the two approaches seem to be rather complementary. The ABM approach is more insightful upon the structure of the economy, while the top-down approach may be more parsimonious in allowing an understanding of the macro interrelationships.

\section{CONCLUDING REMARKS}

This paper has analyzed the dynamics of unemployment in a growth model where both aggregate demand and supply play a fundamental role, while keeping the number of jobless within a significant range. From the demand side, autonomous demand has a driving role in stimulating growth in a medium-run perspective within an economy with slack resources, where wages and prices are given. Furthermore, the presence of a consumption function where heterogeneous agents have different propensity to consume and where income inequality increases with the rate of unemployment, strengthens the loop between aggregate demand and supply rooted in the labor market. In fact, by assuming that the two components of the Harrodian natural rate of growth, i.e. productivity and labor supply, are endogenously determined, not only the interaction between demand and supply is strengthened, but the instability problem is also linked to the so called reconciliation process. This relation can generate a bounded unemployment. Furthermore, in this context, the more flexible are the supply re- 
sponse to aggregate demand dynamics the more the system becomes stable and the dynamics of unemployment remains bounded.

These results, obtained by a top-down approach, are particularly robust both to changes in the values of the parameters and in the specification of the model. Furthermore, the results are robust to changes into expectation hypotheses. For instance, learning has replaced the simple adaptive rule and the results have been strengthened.

These enrichments facilitate the comparison with the results obtained by other contributions, and in particular those referring to the ABM methodology.

The model can be extended in at least three other directions. First, wages and prices must be endogenized. This becomes a priority above all when unemployment is reaching the floor. In the second place, and more in general, monetary and fiscal policies must be considered. These developments would make the comparison with actual data more compelling. Finally, search models of the labor market should also be considered but not in opposition to other determinants of unemployment (see Michaillat, 2012). This is done also by Delli Gatti et al.(2014). In this case, the comparison between the bottom-up methodology vis-à-vis the top-down one becomes even more appealing (see Ferri, 2019).

\section{MATHEMATICAL APPENDIX}

The nonlinear system underlying the analysis is presented in a compact way in what follows. The first equation represents expectations, while the components of aggregate demand are formalized from (A.2) to (A.4). (A.5) represents the evolution of capital, while (A.6) sets the equilibrium in the product market.

(A.7) and (A.8) represent respectively the level and the rate of growth of supply, while (A.8), (A.9), (A.10) and (A.11) show both the levels and the rate of growth of labor supply and productivity. (A.13) defines labor demand, while (A.14) introduces the rate of unemployment. The model is closed by three definitions.

$$
\begin{gathered}
E_{t-1} g_{t}=(1-\alpha) g_{t-1}+\alpha E_{t-2} g_{t-1} \\
C_{t}=\left[Y_{t-1}\left(1+E g_{t}\right)\right]\left[c_{1}-c_{2}\left(u_{t-1}-u_{0}\right)\right]+F_{t} \\
F_{t}=F_{t-1}\left(1+g^{*}\right)\left[1-\psi_{1}\left(u_{t-1}-u_{0}\right)\right] \\
I_{t}=\delta K_{t}+E g_{t} K_{t}+\beta\left[v^{*}\left(1+E g_{t}\right)^{2} Y_{t-1}-\left(1+E g_{t}\right) K_{t}\right]
\end{gathered}
$$




$$
\begin{gathered}
K_{t}=(1-\delta) K_{t-1}+I_{t-1} \\
Y_{t}=C_{t}+I_{t} \\
Y_{t}^{S}=Y_{t-1}^{S}\left(1+g_{t}^{S}\right) \\
g_{t}^{S}=\left(1+\tau_{t}\right)\left(1+\sigma_{t}\right)-1 \\
L_{t}=\left(1+\sigma_{t}\right) L_{t-1} \\
\sigma_{t}=\rho_{0}-\rho_{1} u_{t-1} \\
A_{t}=\left(1+\tau_{t}\right) A_{t-1} \\
\tau_{t}=\theta_{0}-\theta_{1} \frac{i_{t-1}}{v_{t-1}} \\
i_{t}=\frac{I_{t}}{Y_{t}} \\
v_{t}=\frac{Y_{t}}{Y_{t-1}}-1 \\
u_{t}=1-\frac{N_{t}}{L_{t}} \geq 0 \\
N_{t}=\frac{Y_{t}}{A_{t}} \\
\\
v_{t} \\
v_{t} \\
v_{t}
\end{gathered}
$$

Given an exogenous rate of autonomous demand growth $\mathrm{g}^{*}$ and the expected-desired capital-output ratio $v^{*}$, the system refers to 17 unknowns: $I_{t}, K_{t}, C_{t}, F_{t}, Y_{t}, u_{t}, N_{t}, L_{t}, g_{t}$, $v_{t}, \tau_{t}, A_{t}, \sigma_{t}, i_{t}, E g, Y_{t}^{s}$ and $g_{t}^{s}$.

\section{REFERENCES}

Aghion, P. and P. Howitt (1994), 'Growth and unemployment', Review of Economic Studies, 61, 477-494.

Alichi, A., K. Kantenga and J. Solé (2016), 'Income polarization in the United States', IMF Working Paper, No 121, Washington.

Brock, W. and C. Hommes (1997), 'A rational route to randomness', Econometrica, 65, 10591095.

Carrol, C.D. (1992), 'The buffer stock theory of saving: some macroeconomic evidence', Brookings Papers on Economic Activity, 2, 61-156. 
Dawid H. and D. Delli Gatti (2018), 'Agent-based macroeconomics', Bielefeld Working Papers, No 02, Bielefeld.

Delli Gatti, D., E. Desiderio, M. Gaffeo, M. Gallegati and P. Cirillo (2011), Macroeconomics from the Bottom up, Milan, Springer.

Delli Gatti D., M. Gallegati and S. Desiderio (2014), 'The dynamics of the labour market in an agent-based model with financial constraints', in A. Cristini, S.M. Fazzari, E. Greenberg and R. Leoni (eds), Cycles, Growth and The Great Recession, London, Routledge, Chapter 8, 117-129.

Delong, J.B. and L.H. Summers (2012). 'Fiscal policy in a depressed economy', Brookings Papers on Economic Activity, Spring, 233- 274.

De Grauwe, P. (2011), 'Animal spirits and monetary economy', Economic Theory, 47 (2-3),42457.

Dieci, R., and X. He (2018), 'Heterogeneous agent models in finance', in C. Hommes and B. Le Baron (eds), Handbook of Computational Economics, Vol 4, Amsterdam, Elsevier, 257328.

Dosi, G., G. Fagiolo and A. Roventini (2010), 'Schumpeter meeting Keynes. A policy- friendly model of endogenous growth and business cycles', Journal of Economic Dynamics and Control, 34, 1748-1767.

Dosi, G., M. Napolitano, A. Roventini and T. Treibich (2017), 'Micro and macro policies in the Keynes + Schumpeter evolutionary models', Journal of Evolutionary Economics, 27, 63-90.

Dutt, A.K. (2010), 'Reconciling the growth of aggregate demand and aggregate supply', in M. Setterfield (ed), Handbook of Alternative Theories of Economic Growth, Northampton, Edward Elgar, 220-240.

Eusepi, S. and B. Preston (2015), 'Consumption heterogeneity, Employment dynamics and macroeconomic co-movements', Journal of Monetary Economy, 71, 13-32.

Fazzari, S.M., P. Ferri and A.M. Variato (2018), 'Demand-led growth and accommodating Supply', FMM Working Paper, No 15, http://dx.doi.org/10.2139/ssrn.3108711.

Ferri, P. (2011), Macroeconomics of Growth Cycles and Financial Instability, Cheltenham, Edward Elgar.

Ferri, P. (2016), Aggregate Demand, Inequality and Instability, Cheltenham, Edward Elgar.

Ferri, P. (2019), Minsky's Moments: An Insider's View of The Economics of Minsky, Chelthemham, Edward Elgar, forthcoming.

Ferri, P. and A.M. Variato (2010), 'Uncertainty and learning in stochastic macro models', International Advances in Economic Research, 16, 297-310.

Hall, R.E. (2009), 'Reconciling cyclical movements in the marginal value of time and the marginal product of labor', Journal of Political Economy, 117, 281-313.

Hall, R.E. (2017), 'High discounts and high unemployment', American Economic Review, 107, 305-330.

Harrod, R. (1939), 'An essay in dynamic theory', Economic Journal, 49, 14-33. 
Hicks, J.R. (1950), A Contribution to the Theory of the Trade Cycle, Oxford, Oxford University Press.

Goodwin, R.M. (1967), 'A growth cycle', reprinted in 'Essays in Economic Dynamics', (1983) London, Macmillan, Ch. 14.

International Monetary Fund (2018), World Economic Outlook, April, Washington.

Judd, K. and L. Tesfatsion (2006), Handbook of Computational Economics, 2, Agent-Based Computational Economics, Amsterdam, North-Holland.

Kaldor, N. (1957), 'A model of economic growth', Economic Journal, 67, 591-624.

Kaldor, N. (1978), 'Causes of the slow rate of growth in the UK', in Further Essays on Applied Economics, London, Duckworth, vii-xxix.

Lavoie, M. (2014), Post Keynesian Economics: New Foundations, Cheltenham, Edward Elgar.

Malley, J. and T. Moutos (1996), 'Unemployment and consumption', Oxford Economic Papers, $48,584-600$.

McCombie, J. (2002), 'Increasing returns and the Verdoorn law from a Kaldorian perspective'. In J. McCombie, M.Pugno and B. Soro (eds), Essays on Verdoorn's Law, Basingstoke, Palgrave, Mcmillan, 64-114.

Michaillat, P. (2012), 'Do matching frictions explain unemployment? Not in bad times', American Economic Review, 102, 1721-1750.

Minsky, H.P. (1982), 'The integration of simple growth and cycles models', in Can "It" happen again?, New York, Sharpe, 258-277.

Minsky, H.P. (1954/2004), Induced Investment and Business Cycles, Cheltenham, Edward Elgar.

Palley, T.I. (2012), 'Growth, unemployment and endogenous technical progress: A Hicksian resolution of the Harrod's knife-edge', Metroeconomica, 63, 512-541.

Pasinetti, L.L. (1962), 'Rate of profit and income distribution in relation to the rate of economic growth', Review of Economic Studies, 29, 267-279.

Romer, P.M. (1986), 'Increasing returns and long-run growth', Journal of Political Economy, 94, 1002-1037.

Skott, P. (2010), 'Growth, instability and cycles: Harrodian and Kaleckian models of accumulation and income distribution', in Setterfield, M. (ed), Handbook of Alternative Theories of Economic Growth, Northampton, Edward Elgar, 108-141.

Solow, R.M. (1956), 'A contribution to growth theory', Quarterly Journal of Economics, 70, 6594. 